\title{
Temperature Insensitive Strain Sensor with Asymmetrical Cascading Structure Based on Photonic Crystal Fiber in Chemical Corrosion
}

\author{
Guangwei Fu, Yunpu Li, Jiandong Yang, Qifeng Li, Xinghu Fu, and Weihong Bi \\ School of Information Science and Engineering, The Key Laboratory for Special Fiber and Fiber Sensor of Hebei Province, \\ Yanshan University, Qinhuangdao 066004, China \\ Correspondence should be addressed to Guangwei Fu; earl@ysu.edu.cn and Weihong Bi; whbi@ysu.edu.cn
}

Received 19 July 2017; Revised 7 September 2017; Accepted 11 September 2017; Published 19 November 2017

Academic Editor: Lei Yuan

Copyright (c) 2017 Guangwei Fu et al. This is an open access article distributed under the Creative Commons Attribution License, which permits unrestricted use, distribution, and reproduction in any medium, provided the original work is properly cited.

\begin{abstract}
In this paper, a corroded photonic crystal fiber (PCF) sensor is presented on the basis of core-offset splicing and waist-enlarged fiber taper corroded by hydrofluoric acid (HF). The Mach-Zehnder interference theory is used to analyze the relationship between transmission spectrum and strain. The final results obtained from experiments show that the strain sensor's sensitivity is about $-6.57 \mathrm{pm} / \mu \varepsilon$ and the linearity is 0.99962 within the range of $0 \sim 1000 \mu \varepsilon$, while the sensor is corroded by HF for 35 minutes at room temperature. In the temperature range of $20^{\circ} \mathrm{C} \sim 80^{\circ} \mathrm{C}$, the sensor's sensitivity to temperature is only $1.63 \mathrm{pm} /{ }^{\circ} \mathrm{C}$, which is insensitive to temperature. Because of its advantage compared to traditional optical fiber sensors, this sensor can avert the cross-sensitive problem when temperature and strain are measured together and has some other advantages including easily fabricated, simple structure and accurate sensitivity. It can be used in many fields, such as industrial production, monitoring, and aerospace.
\end{abstract}

\section{Introduction}

With the development of science and technology, the requirements for the safety monitoring of large-scale buildings and the sensitivity of high precision instruments are getting higher and higher. Therefore, the corresponding requirements for strain change are higher. Compared with the traditional sensor, the optical fiber sensor has the advantages of small volume, lightweight, corrosion resistance, and antielectromagnetic interference [1-4]. Thus its application is more and more extensive, especially for measurement of temperature [5], strain [6], refractive index [7], and other parameters. Osuch et al. [8] ever reported a sensor using linearly chirped tapered fiber Bragg gratings to measure the strain. The strain sensitivity is $4.7 \mathrm{pm} / \mu \varepsilon$. Sun et al. [9] fitted the thin-core fiber into a single mode fiber showed a strain sensor, structure with thin-core fiber inserted into a standard single mode fiber, and completed the simultaneous measurement of strain and temperature with the recording sensitivity of $1.83 \mathrm{pm} / \mu \varepsilon$ to strain and of $72.89 \mathrm{pm} /{ }^{\circ} \mathrm{C}$ to temperature on the foundation of Mach-Zehnder principle. Moreover, Noh et al. [4] applied photonic crystal fiber to a Sagnac birefringence loop and got a sensitivity of $1.91 \mathrm{pm} / \mu \varepsilon$. However, all the sensors mentioned above have the problems that the sensitivity is not very high and the simultaneous measurement of the two parameters is cross-sensitive.

Therefore, in order to improve strain sensitivity of the sensor, a corrosion-type strain sensor of core-offset splicing and waist-enlarged photonic crystal fiber [10] (co\&we-PCF) is presented. The sensor is prepared by corroding a section of the optical fiber between the two joints, core-offset joint, and excessive fusion joint, stretched in HF solution. The sensor with different fiber diameter is fabricated by controlling the corrosion time of the fiber in HF; then a corrosive co\&wePCF strain sensor is obtained. The strain featured sensor is studied by experiment.

\section{Theory Analysis}

Co\&we-PCF could be obtained by using the method reported in [10]. The construction of the corroded PCF strain sensor 


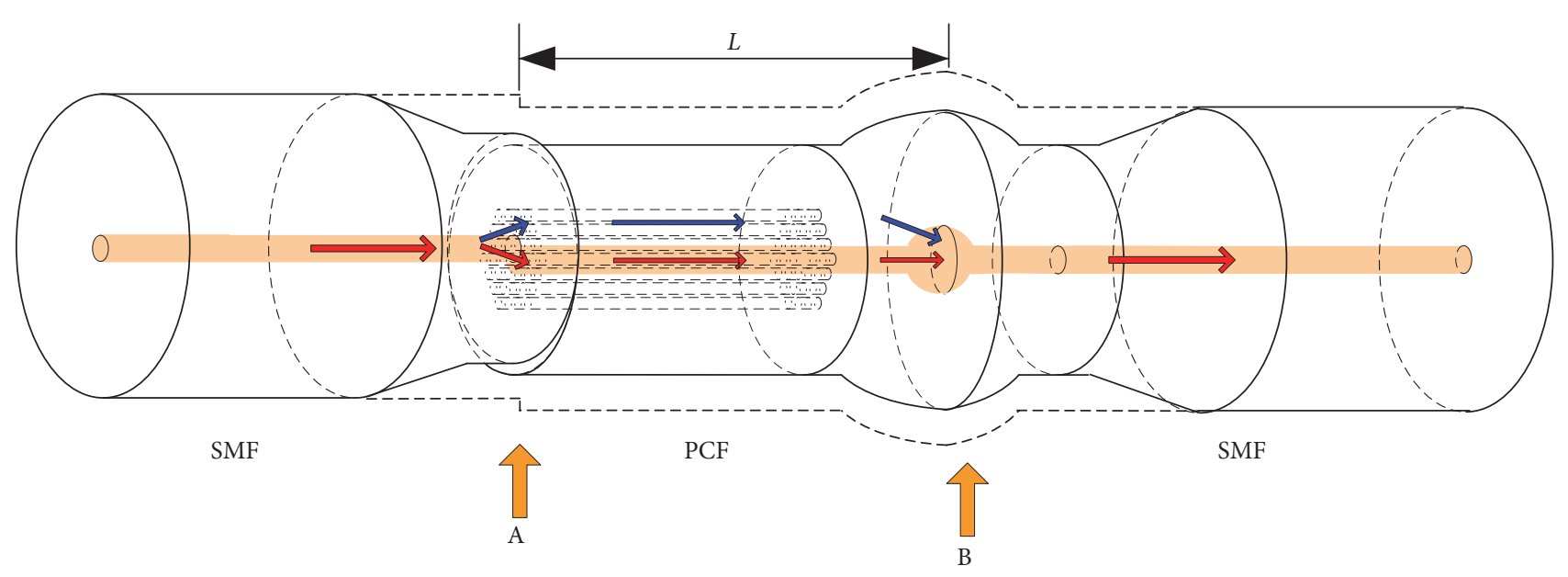

Figure 1: The structure of sensor.

is shown in Figure 1. It has an asymmetrical corrosive SMFPCF-SMF structure.

As shown in Figure 1, $L$ is the fusion length of PCF, A and $\mathrm{B}$ are the core-offset joint and the excessive fusion joint, respectively. When light enters into SMF, most of the light energy is bounded and transferred into the fiber core. Then the light passes through joint $\mathrm{A}$, and the optical fiber structure changes due to the core-offset splicing, which causes fiber mode mismatch. At the same time, some light pierces the clad of PCF. Because the effective refractive index of cladding mode and the core mode are different, there is a phase difference after a certain distance of light transmission. Then the two parts of the light interfere at joint B and form MachZehnder interferometer. So the intensity of the sensor output light [11-13] is

$$
\begin{aligned}
& I=I_{\text {core }}+I_{\text {clad }}+2 \sqrt{I_{\text {core }} I_{\text {clad }}} \cos \varphi, \\
& \varphi=\frac{2 \pi\left(n_{\text {eff }}^{\text {core }}-n_{\text {eff }}^{\text {clad }}\right) L}{\lambda},
\end{aligned}
$$

where $I_{\text {core }}$ is the intensity of fiber core mode, $I_{\text {clad }}$ is the intensity of the cladding mode, $n_{\mathrm{eff}}^{\text {core }}$ is the effective refractive index of fiber core mode, and $n_{\mathrm{eff}}^{\mathrm{clad}}$ is the effective refractive index of cladding mode. $\lambda$ is the wavelength of optical transmission and $L$ is the fusion splicing length of PCF. During the light transmission process, the interference spectrum will change periodically. Thus variations of peaks and troughs appear. The peaks will appear at $2 m \pi$. Therefore, the wavelength of the max interference center of the $m$ order of the interference spectrum can be expressed as follows [14]:

$$
\lambda_{m}=\frac{\left(n_{\mathrm{eff}}^{\text {core }}-n_{\mathrm{eff}}^{\text {clad }}\right) L}{m} .
$$

The central wavelength drift of the $m$ order interference is

$$
\Delta \lambda_{m}=\left(1+p_{s}\right) \lambda_{m} \varepsilon
$$

where the strain response factor is

$$
p_{s}=\frac{\left(D / \Delta n_{\mathrm{eff}}\right) \partial\left(n_{\mathrm{eff}}^{\mathrm{core}}-n_{\mathrm{eff}}^{\mathrm{clad}}\right)}{\partial D} .
$$

Here the strain is

$$
\varepsilon=\frac{\Delta D}{D}
$$

where $\Delta D$ is the moving distance of platform 2 (the platform 2 is marked in Figure 3) along axial direction, and it moves $0.01 \mathrm{~mm}$ every time. $D$ is the initial distance between the two platforms, which is set as $10 \mathrm{~cm}$.

From (3), (4), and (5), the strain response factor can be expressed:

$$
p_{s}=\frac{\left(1 /\left(n_{\mathrm{eff}}^{\text {core }}-n_{\mathrm{eff}}^{\text {clad }}\right)\right) \partial\left(n_{\mathrm{eff}}^{\text {core }}-n_{\mathrm{eff}}^{\text {clad }}\right)}{\partial \varepsilon} .
$$

So the magnitude of effective refractive index difference between the core and cladding modes and strain can affect the wavelength drift of the $m$ order interference fringes, but $\Delta \lambda_{m}$ is not related to the length of the PCF. And the wavelength drift of the spectra is proportional to the magnitude of the strain. The wavelength will occur to drift while the strain increases.

\section{Experimental Results}

3.1. Sensors Fabrication. Corrosion-type PCF sensor is prepared by core-offset splicing SMF and PCF firstly and then excessive splicing PCF and SMF on fusion splicer. Corrode the optical fiber between A and B into HF. The sensor with different fiber diameter is obtained by controlling the corrosion time at the corrosion rate of $2 \mu \mathrm{m} / \mathrm{min}$ in the experiment. A sensor with PCF fusion length of $20 \mathrm{~mm}$, offset of $7 \mu \mathrm{m}$, and taper diameter of $160 \mathrm{~mm}$ is corroded in HF for $35 \mathrm{~min}$ and then is washed by distilled water to clean the residual HF on sensor. The micrographs of the sensor are shown in Figure 2.

In Figure 2, after hydrofluoric acid corrosion with $35 \mathrm{~min}$, the sensor with PCF diameter of $57.46 \mu \mathrm{m}$ offset is still $7 \mu \mathrm{m}$ 


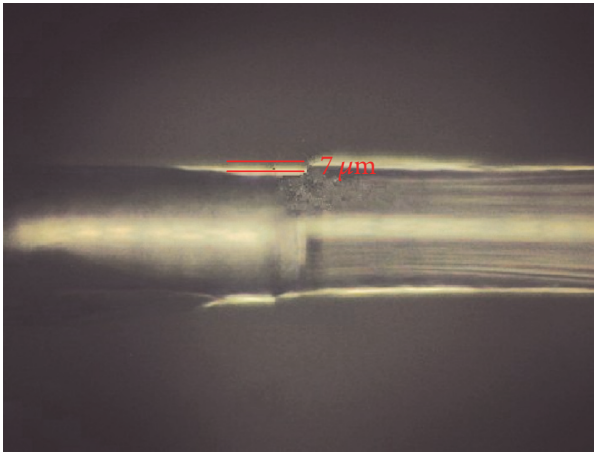

(a)

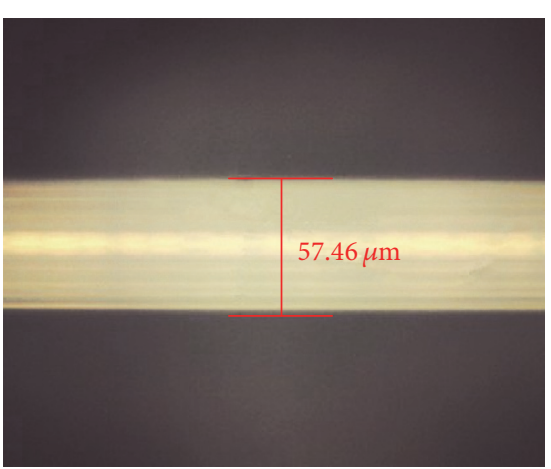

(b)

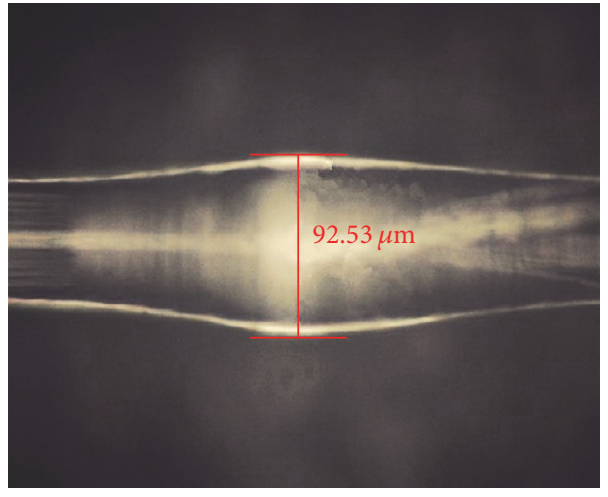

(c)

FIGURE 2: (a) Micrographs of corrosive fiber with core-offset splicing. (b) Micrographs of fusion splicing length of PCF after being corroded. (c) Micrographs of excessive splicing waist-enlarged fiber taper after being corroded.

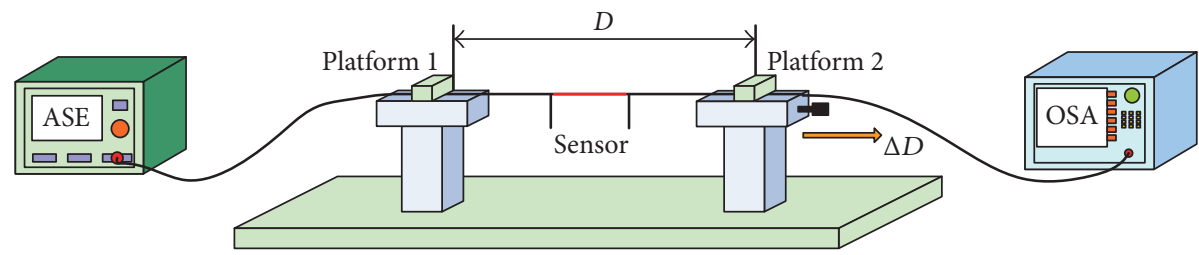

FIGURE 3: Strain measurement experiment system.

but taper diameter becomes $92.53 \mu \mathrm{m}$. Because both sides of the core-offset splicing joint are corroded at the same time, the offset length still remains $7 \mu \mathrm{m}$. Since HF diffuses slightly over time, the spliced joints of SMF corroded a portion unavoidably, so that a gentle slope is formed between fusion joints and lead-in and lead-out SMF, respectively. The reasons for choosing this structure are as follows: core-offset splicing SMF and PCF can improve optical path difference, which is conducive to the formation of the $\mathrm{M}-\mathrm{Z}$ interference; excessive splicing PCF and SMF can cause the light in core and clad interference and effectively expand the measurement range of strain; corroding the optical fiber between A and B can increase evanescent field, which makes the corroded fiber optic sensor more sensitive.

3.2. Experiment Process. Figure 3 shows the schematic diagram of the strain measurement experiment system. The sensors are connected to the Amplified Spontaneous Emission (ASE) broadband light source and optical spectrum analyzer (OSA), respectively. In the sensing experiment, the ASE broadband light source wavelength ranges from $1520 \mathrm{~nm}$ to $1610 \mathrm{~nm}$, and the optical spectrum analyzer (AQ6375) is used to record experimental data. Figure 3 shows the schematic diagram of strain measurement.

In the experiment, the prepared sensor is put firmly on platforms 1 and 2 in a stretched state, and the strain is changed by moving platform 2 . The initial fixed distance between the two platforms is set at $10 \mathrm{~cm}$; each time platform 2 moves to the right $0.01 \mathrm{~mm}$. By (5), the strain of the sensor will be increased by $100 \mu \varepsilon$ at every stretch of the sensor each time. Platform 2 moves to the right with a total of 10 times and the transmission spectrum is recorded every time. So the strain sensing experiments of the sensor are done within the range of $0 \sim 1000 \mu \varepsilon$. Different strain transmitted spectrum 


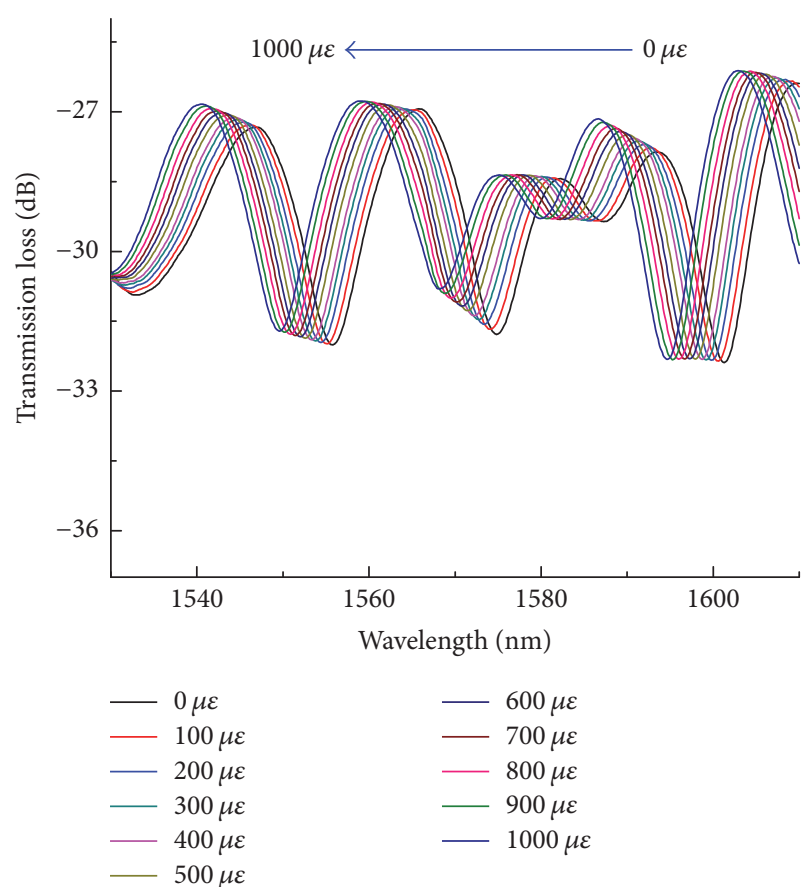

(a)

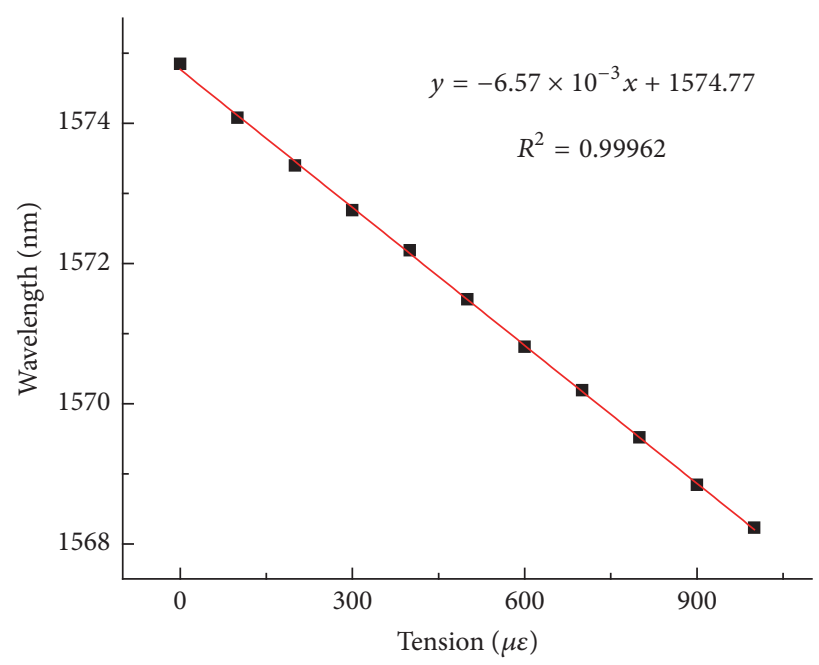

(b)

Figure 4: (a) The transmission spectrum of different strain. (b) The liner dependence relation of strain and wavelength shift.

TABLE 1: Strain sensitivity of different length.

\begin{tabular}{lc}
\hline Corroding time & sensitivity \\
\hline $10 \mathrm{~min}$ & $-2.14 \mathrm{pm} / \mu \varepsilon$ \\
$20 \mathrm{~min}$ & $-3.4 \mathrm{pm} / \mu \varepsilon$ \\
$30 \mathrm{~min}$ & $-5.5 \mathrm{pm} / \mu \varepsilon$ \\
$35 \mathrm{~min}$ & $-6.57 \mathrm{pm} / \mu \varepsilon$ \\
\hline
\end{tabular}

and its linear dependence relation with wavelength drift corresponding to the wave trough near $1570 \mathrm{~nm}$ are shown in Figure 4.

It can be seen from Figure 4 that the transmitted spectra drift to the short wave direction as the strain increases. In the range of $0 \sim 1000 \mu \varepsilon$, from the figured out shift of the transmitted spectra near $1570 \mathrm{~nm}$ trough, we can obviously know the sensitivity is $-6.57 \mathrm{pm} / \mu \varepsilon$ and the linearity is 0.99962 . The wavelength drift has a good linear respondence relation with the strain varying. Through controlling the corrosion time of $10 \mathrm{~min}, 20 \mathrm{~min}, 30 \mathrm{~min}$, and $35 \mathrm{~min}$, respectively, different fiber diameter of the sensor should be investigated in the experiment. The experimental results are present in Table 1.

From the table, we can know that the strain sensitivity will improve with corroding time increasing. As for different fiber diameter corrosion PCF sensor, the sensitivity will increase as the diameter of the fiber decreases. But when corrosive time is over $35 \mathrm{~min}$, the air holes of PCF are corroded. The sensitivity of the sensor is maximum when the corrosion time is $35 \mathrm{~min}$. As the fiber cladding of the sensor is corroded, the evanescent field and the intensity of the transmitted light in the fiber

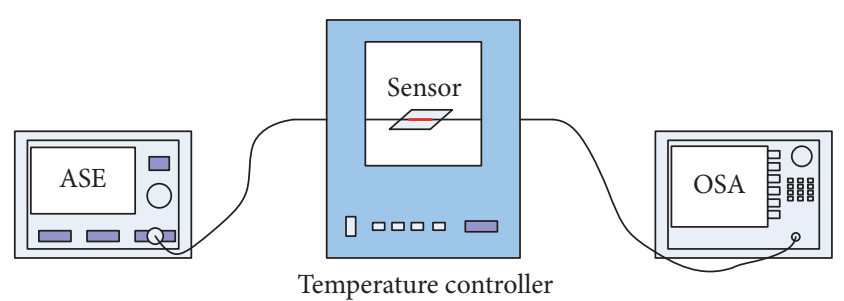

FIGURE 5: Temperature measurement experiment system.

cladding increase. And the diameter of the cladding decreases resulting in the distance between the core and the edge of the cladding reduced. The elastooptical effect is stronger for the light closer to the edge of the cladding, which makes the corroded fiber optic sensor sensitive to the strain.

Cao et al. [15] measured the strain sensing by a photosensitive fiber (PSF) core-offset splicing structure sensor and gained a sensitivity of $-0.64 \mathrm{pm} / \mu \varepsilon$. Xu et al. [16] used the SMF-PCF-SMF structure to measure the strain and got the sensitivity of $3.02 \mathrm{pm} / \mu \varepsilon$. Compared with other co\&we-PCF, the method and structure of the sensor proposed in this paper are simple and the sensitivity is also improved.

3.3. Temperature Sensing Experiment. In the temperature sensing experiment, fix the prepared sensor on the glass plate and then place it in the temperature control box. Connect one end of the sensor to ASE light source and another to OSA. Experimental schematic diagram is shown in Figure 5.

Then measure spectrum at different temperature. The temperature changes are set in the range of $20 \sim 80^{\circ} \mathrm{C}$ on the 


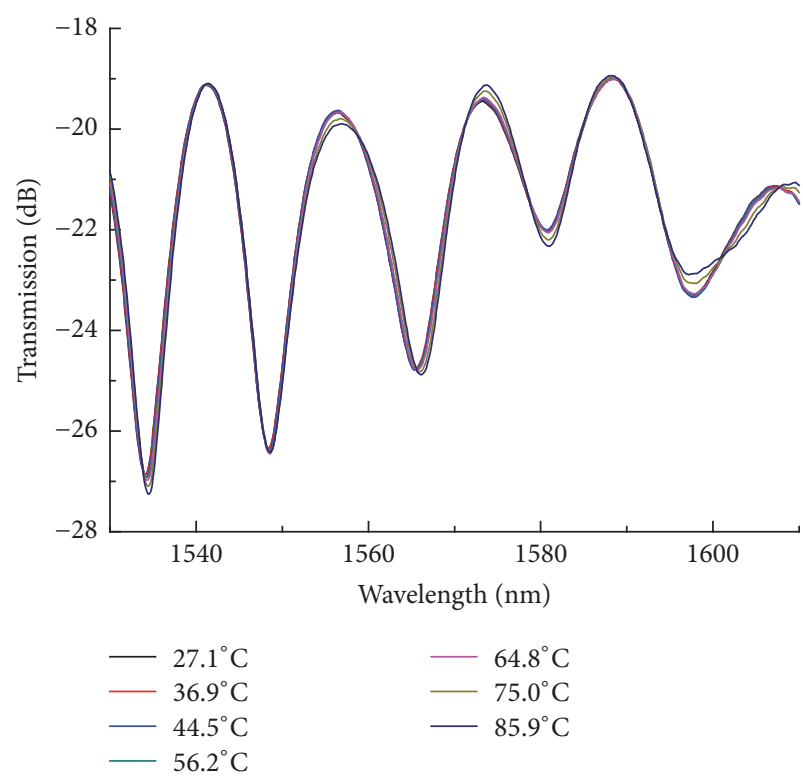

(a)

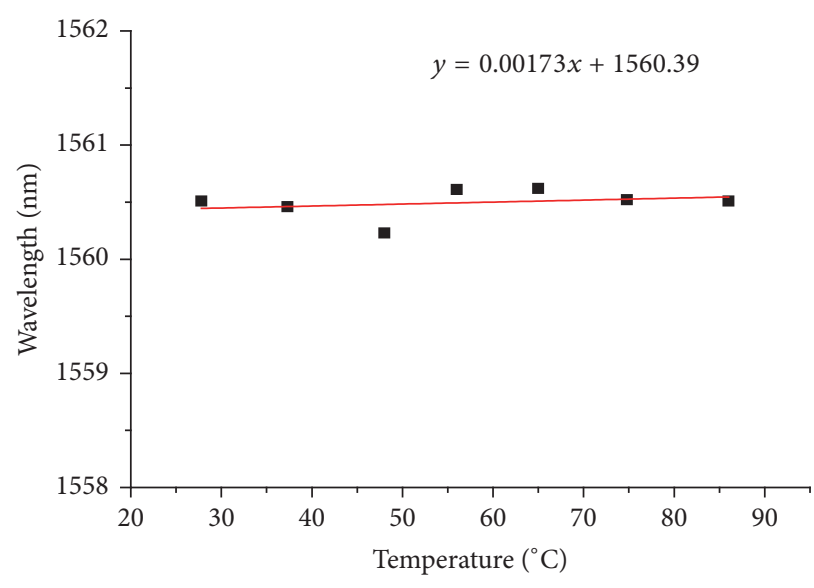

(b)

FIGURE 6: (a) The transmission spectra of different temperature. (b) The relationship between temperature and wavelength shift.

temperature control box. When the temperature increases approximately $10^{\circ} \mathrm{C}$ and stays stable, the spectrum is recorded at that time. The spectra corresponding to different temperatures are shown in Figure 6.

Figure 6 shows that the transmitted spectrum has a slight shift to long wave with temperature increase. The relationship between wavelength shift and temperature is obtained by analyzing the transmitted spectrum and calculating the shift of wavelength near $1560 \mathrm{~nm}$ trough. As shown in Figure 6(b), its temperature sensitivity is just $1.73 \mathrm{pm} /{ }^{\circ} \mathrm{C}$, hinting its insensitivity to temperature. That is, when temperature changes every degree, the wavelength shift changes $1.73 \mathrm{pm}$. In particular, these experiments are done at room temperature with minute changes, and the wavelength shift is tiny compared to that of strain measurements. Thereby, we can ignore the temperature influence when working on strain sensing. It can be considered that the sensor is insensitive to temperature.

\section{Conclusion}

In this paper, a corrosion-type co\&we-PCF taper strain sensor is proposed. It is fabricated by core-offset splicing and excessively splicing a $20 \mathrm{~mm}$ PCF with SMFs at the two ends, respectively, and then corroded in HF for 35 minutes. The experimental results show that the linear sensitivity near $1570 \mathrm{~nm}$ trough is $-6.57 \mathrm{pm} / \mu \varepsilon$ and the linearity is 0.99962 . The wavelength drift has a good linear dependence relation with the strain change. And the cross-sensitive problems of temperature and strain can be avoided because the sensor is not sensitive to temperature. The PCF sensor presented in this paper has the characteristic of high sensitivity and simple structure and is easily fabricated. It has a good application in the accurate measurement of strain in bridge, construction, aerospace, and so on.

\section{Conflicts of Interest}

The authors declare that there are no conflicts of interest regarding the publication of this paper.

\section{Acknowledgments}

This project is supported by the National Natural Science Foundation of China (nos. 61575170, 61475133, and 61675176), the Key Applied Basic Research Program of Hebei Province (nos. 16961701D and QN2016078), Hebei Provincial Natural Science Foundation (nos. F2015203270 and F2016203392), Qinhuangdao Science and Technology Support Program (no. 201601B050), College Youth Talent Project of Hebei Province (no. BJ2014057), and "XinRuiGongCheng" Talent Project of Yanshan University.

\section{References}

[1] H. H. Qazi, A. B. Mohammad, H. Ahmad, and M. Z. Zulkifli, "D-shaped polarization maintaining fiber sensor for strain and temperature monitoring," Sensors, vol. 16, no. 9, article 1505, 2016.

[2] L. M. Hu, C. C. Chan, X. Y. Dong et al., "Photonic crystal fiber strain sensor based on modified mach-zehnder interferometer," IEEE Photonics Journal, vol. 4, no. 1, pp. 114-118, 2012.

[3] G. Fu, Q. Li, Y. Li et al., "The tension sensor of Photonic Crystal Fiber based on core-offset splicing and waist-enlarged fiber taper," in Proceedings of the International Symposium on Optoelectronic Technology and Application, vol. 10158, Beijing, China, May 2016.

[4] T. K. Noh, S.-M. Lee, E.-S. Kim, B.-S. Shin, and Y. W. Lee, "Photonic-crystal-fiber-based temperature-insensitive polarimetric fiber strain sensor with reduced length of sensing fiber," in Proceedings of the 17th Opto-Electronics and Communications 
Conference (OECC '12), pp. 371-372, Busan, South Korea, July 2012.

[5] J. N. Dash and R. Jha, "Mach-Zehnder interferometer based on tapered PCF with an up-tapered joint for curvature, strain and temperature interrogation," Journal of Optics, vol. 18, no. 10, Article ID 105002, 2016.

[6] H. Sun, S. Yang, X. Zhang, L. Yuan, Z. Yang, and M. Hu, "Simultaneous measurement of temperature and strain or temperature and curvature based on an optical fiber Mach-Zehnder interferometer," Optics Communications, vol. 340, pp. 39-43, 2015.

[7] X. Sun, D. Dai, L. Thylén, and L. Wosinski, "High-sensitivity liquid refractive-index sensor based on a Mach-Zehnder interferometer with a double-slot hybrid plasmonic waveguide," Optics Express, vol. 23, no. 20, pp. 25688-25699, 2015.

[8] T. Osuch, K. Markowski, and K. Jedrzejewski, "Fiber-optic strain sensors based on linearly chirped tapered fiber Bragg gratings with tailored intrinsic chirp," IEEE Sensors Journal, vol. 16, no. 20, pp. 7508-7514, 2016.

[9] M. Sun, B. Xu, X. Dong, and Y. Li, "Optical fiber strain and temperature sensor based on an in-line Mach-Zehnder interferometer using thin-core fiber," Optics Communications, vol. 285, no. 18, pp. 3721-3725, 2012.

[10] G. Fu, Y. Li, Q. Li, J. Yang, X. Fu, and W. Bi, “Temperature insensitive vector bending sensor based on asymmetrical cascading SMF-PCF-SMF structure," IEEE Photonics Journal, vol. 9, no. 3, pp. 1-14, 2017.

[11] H. F. Martins, J. Bierlich, K. Wondraczek et al., "High-sensitivity dispersive Mach-Zehnder interferometer based on a dissimilardoping dual-core fiber for sensing applications," Optics Expresss, vol. 39, no. 9, pp. 2763-2766, 2014.

[12] Y. Zhang, Y. Yu, C. Du et al., "Strain-independent high-temperature sensor with a suspended-core fiber based Mach-Zehnder interferometer," Optical Fiber Technology, vol. 29, pp. 6-10, 2016.

[13] D. Wu, Y. Zhao, and J. Li, "PCF taper-based Mach-Zehnder interferometer for refractive index sensing in a PDMS detection cell," Sensors and Actuators B: Chemical, vol. 213, pp. 1-4, 2015.

[14] D. Wu, T. Zhu, M. Deng et al., "Refractive index sensing based on Mach-Zehnder interferometer formed by three cascaded single-mode fiber tapers," Applied Optics, vol. 50, no. 11, pp. 1548-1553, 2011.

[15] Z. Cao, X. Ji, R. Wang et al., "Compact fiber sensor with high spatial resolution for simultaneous strain and temperature measurement," IEEE Sensors Journal, vol. 13, no. 5, pp. 1447-1451, 2013.

[16] F. Xu, C. Li, D. Ren et al., “Temperature-insensitive MachZehnder interferometric strain sensor based on concatenating two waist-enlarged fiber tapers," Chinese Optics Letters, vol. 10, no. 7, Article ID 070603, 2012. 


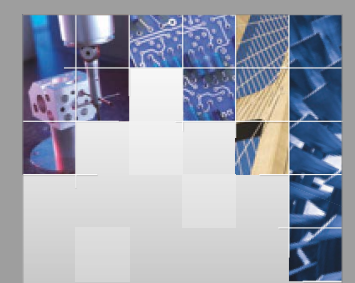

\section{Enfincering}
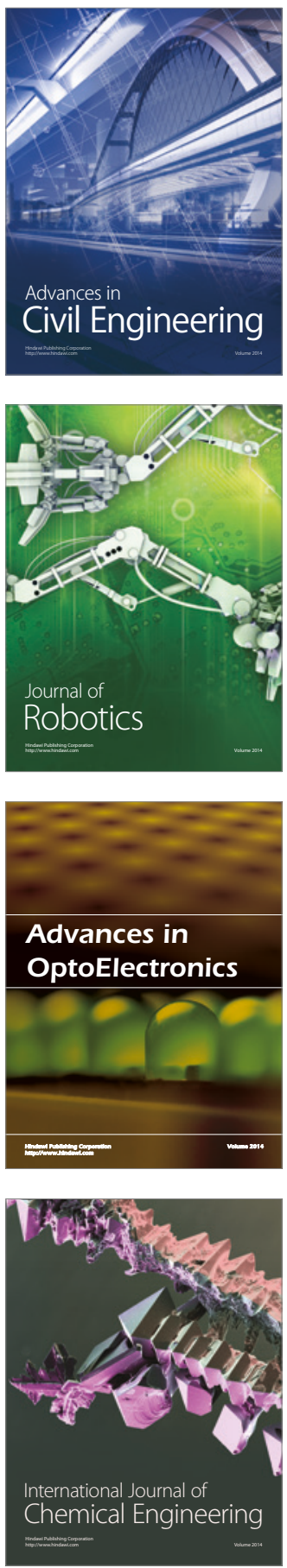

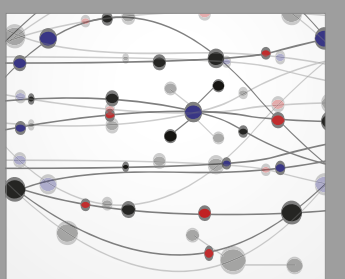

The Scientific World Journal

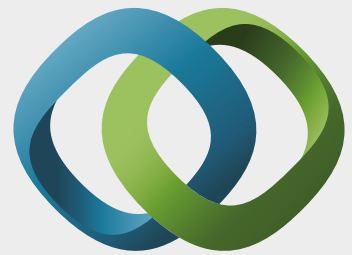

\section{Hindawi}

Submit your manuscripts at

https://www.hindawi.com
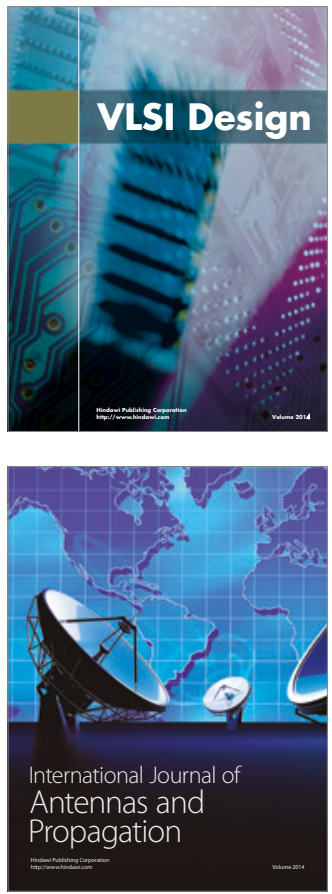

\section{Rotating}

Machinery
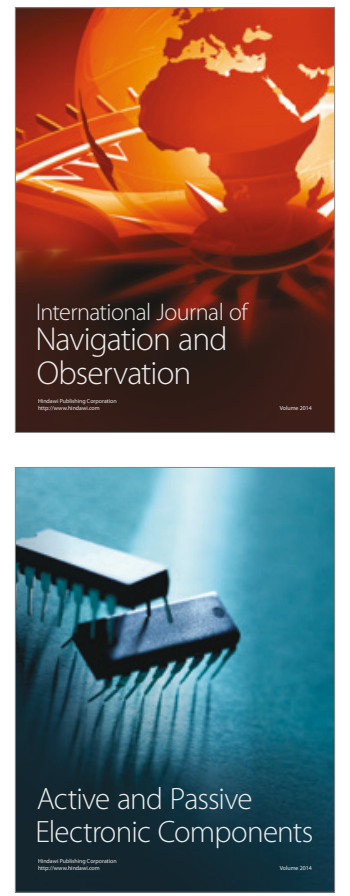
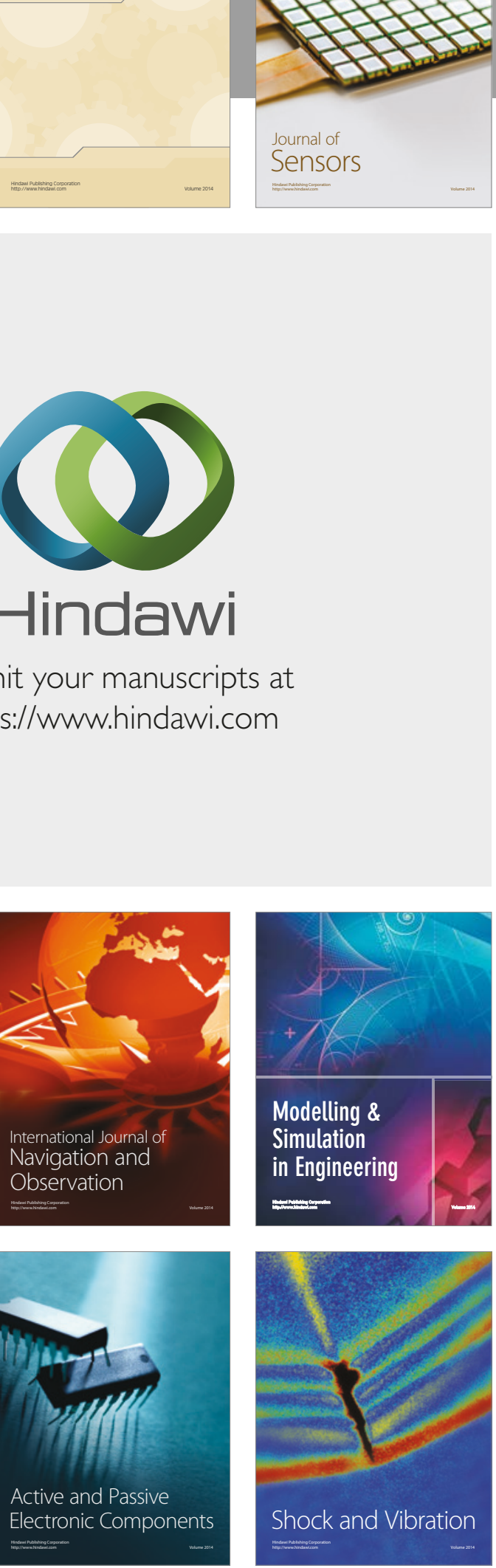
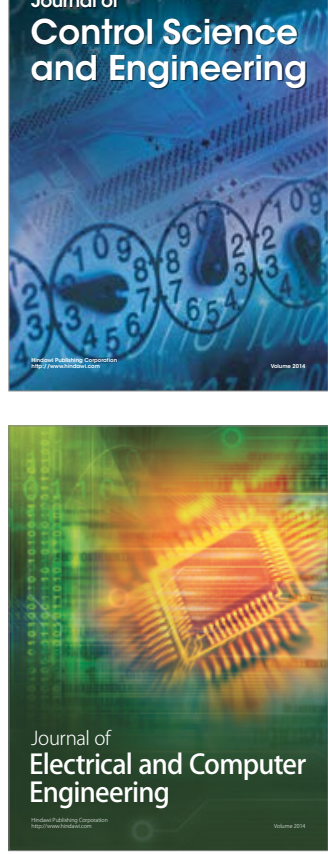

Distributed

Journal of

Control Science

and Engineering
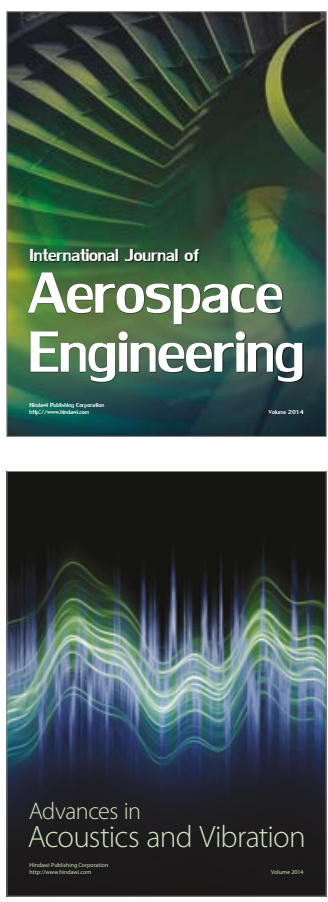

Sensor Networks 